\title{
Foundational ontologies meet ontology matching: A survey
}

\author{
Cassia Trojahn ${ }^{\text {a }}$, Renata Vieira ${ }^{\mathrm{b}}$, Daniela Schmidt ${ }^{\mathrm{c}}$, Adam Pease $^{\mathrm{d}}$ and Giancarlo Guizzardi e,f,* \\ ${ }^{a}$ Institut de Recherche en Informatique de Toulouse, France \\ ${ }^{\mathrm{b}}$ CIDEHUS, University of Évora, Portugal \\ ${ }^{\mathrm{c}}$ School of Technology, PUCRS, Brazil \\ ${ }^{\mathrm{d}}$ Articulate Software, USA \\ e Free University of Bozen-Bolzano, Italy \\ ${ }^{\mathrm{f}}$ University of Twente, The Netherlands
}

Editor: Jérôme Euzenat, INRIA Grenoble Rhône-Alpes, France

Solicited reviews: Michael Uschold, Semantic Arts, USA; Four anonymous reviewers

\begin{abstract}
Ontology matching is a research area aimed at finding ways to make different ontologies interoperable. Solutions to the problem have been proposed from different disciplines, including databases, natural language processing, and machine learning. The role of foundational ontologies for ontology matching is an important one, as they provide a well-founded reference model that can be shared across domains. It is multifaceted and with room for development. This paper presents an overview of the different tasks involved in ontology matching that consider foundational ontologies. We discuss the strengths and weaknesses of existing proposals and highlight the challenges to be addressed in the future.
\end{abstract}

Keywords: Ontology matching, fondational ontologies, lexicon matching, survey

\section{Introduction}

A primary purpose of work on ontology is to create a common computable semantics for concepts in the world. An ontology describes the concepts and the relation between these concepts together with constraints on how to interpret them. Foundational ontologies aim to create a computable semantics for very general concepts and associated terms. With a large number of ontologies having been created for different domains, as well as different conceptualizations for upper, top or foundational ontologies, the creation of shared semantic descriptions is still an issue.

The role of fondational ontologies in ontology construction, matching and integration is manifold. Their potential for clarity in semantics and a rich formalization are important requirements for ontology development improving ontology quality [41,52] and preventing bad ontology design [34,75]. These ontologies may also act as semantic bridges supporting interoperability between ontologies [35,50,51].

In the semantic web and linked data in general, as stated in [3], distinctions such as whether an entity is inherently a class or an individual, or whether it is a physical object or not, are rarely expressed in the linked data, although they have been largely studied and formalised by foundational ontologies. As stated by the authors, while

*Corresponding author. E-mail: Giancarlo.Guizzardi@unibz.it.

1570-0844 (C) 2022 - The authors. Published by IOS Press. This is an Open Access article distributed under the terms of the Creative Commons Attribution License (CC BY 4.0). 
the first distinction showed its practical importance in modelling and meta-modelling approaches, it is also at the basis of knowledge representation formalisms (such as RDF and OWL) for supporting taxonomic reasoning. The second distinction (physical object or not) is essential to represent the physical world and express their expected physical behaviour (e.g., spatial extension, gravity). Such distinctions are however nearly ubiquitous in ontologies and domain data described by these ontologies.

When a foundational ontology is used in the development and integration of domain ontologies - during or after top-down and bottom-up approaches may be considered [76]. With a top-down approach, the foundational ontology is used as a reference for deriving domain concepts, taking advantage of the knowledge and experience already encoded in it. In a bottom-up approach, one usually matches an existing domain ontology to a foundational one. The latter is more challenging since inconsistencies may exist between domain and foundational ontologies. For example, a domain ontology might be about hypothetical or fictional entities, and a particular foundational ontology might commit only to real entities. Furthermore, one has to deal with different levels of abstraction and also of formalization in the matching process.

Ontology matching is a research area aimed at finding ways to make different ontologies interoperable. The matching process can be seen as the task of generating a set of correspondences (i.e., an alignment) between the entities of different ontologies [18]. Correspondences express relationships between ontology entities. For instance, the concept of Author in one source ontology may be marked as equivalent (that can be interpreted as logical equivalence or more informally as a high level of similarity) to the concept of Writer in one target ontology, or that Writer in the source is subclass of Person in the target. One's intuitions about the formalizations of those words may be that they are different, but unless an ontology specifies a formalized definition, they are just labels. A linguistic-based matching algorithm might find them sufficiently similar and evaluators of the match would not have an objective basis for declaring it faulty.

Whereas the area of ontology matching has developed in the last decades, the problem of matching ontologies involving foundational ontologies has seen less development regarding automatic solutions [44,70]. This is not surprising since matching foundational and domain ontologies is a highly complex task, even when done manually. It requires the deep identification of the semantic context of concepts and, at a minimum, the identification of subsumption relations, and in a way such that the subsumption relations must of course be consistent with the formalization of the subsuming concept in the upper ontology. In fact, subsumption and other relations are often neglected by most state-of-the-art matchers.

There have been many manual efforts to make sense of how different foundational ontologies relate to other lexical and semantic data bases, and how they improve the process of matching domain ontologies. In this paper, we survey various approaches to ontology matching using foundational ontologies to create shared semantics.

An additional challenge is that there is little agreement on many of the possible goals and methods of ontology construction or the formal languages in which to encode an ontology. Developers of ontologies have variously advocated very small upper ontologies or large ones, very expressive formal logics, or very minimal ones as a way to support fast logical inference. Evaluations and surveys have typically been conducted by the authors themselves, or their collaborators and supporters (with the possible exception of [50]).

Considering this scenario, this paper reviews the following tasks of ontology matching involving foundational ontologies:

(i) matching of foundational ontologies;

(ii) matching of foundational ontologies to lexicons;

(iii) matching domain ontologies with the help of foundational ontologies; and

(iv) matching foundational ontologies to domain ontologies.

We discuss the main strengths and weaknesses of existing approaches and highlight the challenges to be addressed in the future. We consider that this comprehensive study may set the grounds for advancing domain and foundational ontology matching.

The scope of this paper is in using foundational ontologies for matching and integration of ontologies. While this necessarily touches on the topics of how to create or evaluate ontologies themselves, we will address this topic only in the service of evaluation the use case of ontologies for matching. As such, we do not attempt to review all available foundational ontologies, but just study the use of them for matching other ontologies. 
The rest of the paper is organised as follows: Section 2 introduces the different foundational ontologies and ontology matching. Section 3-Section 6 discuss the approaches in the categories (i)-(iv) introduced above. Section 7 discusses the open challenges in the field and Section 8 concludes the survey.

\section{Background}

\subsection{Foundational ontologies}

An ontology typically provides a vocabulary that describes a domain of interest and a specification of the meaning of terms used in the vocabulary. Depending on the precision of this specification, the notion of ontology encompasses several data and conceptual models, for example, sets of terms, classifications, database schemes, or fully axiomatized theories [80].

In particular, ontologies can be classified according to their "level of generality" [30]: (i) foundational ontologies describe general concepts (e.g., object, event, quality) and relations (e.g., parthood, participation, dependence, causality), which are independent of a particular domain. These ontologies, also called upper or top-level, are sometimes equipped with detailed logical formalizations, and some include (ii) domain ontologies that may also describe the entities related to a particular domain (e.g., biology or aeronautics). ${ }^{1}$ A foundational ontology is often characterized as representing commonsense concepts and is focused on concepts that are meta, generic, and domainindependent.

Several foundational ontologies have been developed, influenced by different philosophies and views on how to conceptualize reality. Several comparisons can be found in the literature, as in $[43,50,76]$. Some common criteria for comparing ontologies are artifact representation criteria (dimensions, representation languages, modularity) [50], ontological commitments and subject domain and applications [43].

We list a number of well-known ontologies that have documented use in ontology matching and integration efforts. We introduce the main insights behind each proposal, and current term (class+property) and axiom counts. Different variants and versions, and the availability of alignments to lexical resources (such as WordNet [54]) and ontologies are discussed in the next sections.

- BFO $[2,27]^{2}$ (Basic Formal Ontology) adopts a realistic approach in terms of the existence in time of entities populating the world. It represents the reality into two disjoint categories of continuant (independent and dependent continuants, attributes, and locations) and occurrent (processes and temporal regions). It has 35 terms and a similar number of axioms. It is defined in $\mathrm{OWL}^{3}$ and first-order logic language CLIF. ${ }^{4}$

- DOLCE [21] (Descriptive Ontology for Linguistic and Cognitive Engineering) is an ontology of particulars which adopts a descriptive approach. It is based on a fundamental distinction between endurant and perdurant entities. Endurants represent objects or substances while perdurants corresponds to events or processes. The main relation between endurants and perdurants is that of participation. DOLCE was originally written in the first-order logical language KIF [26] and includes roughly 100 terms and a similar number of axioms. Recent work maintains DOLCE in OWL.

- Cyc [32] is a proprietary ontology comprising both an upper-level ontology and a set of domain ontologies in a wide variety of domains. It is meant for the representation of facts, rules, and heuristics to reason about the objects and events of everyday life in the Cyc knowledge base. It involves thousands of "microtheories" with hundreds of thousands of terms and millions of axioms. It comprises OpenCyc is an open source subset of Cyc that is no longer maintained. It is defined in the higher-order CycL language [46].

\footnotetext{
${ }^{1}$ We here follow the terminology proposed by [30] in distinguishing top-level and domain ontologies regarding their level of generality. More recently, the term Core Ontology [15,68] has been used to refer to ontologies whose generality level lays half-way between top-level and domain ontologies. These can be seen as abstractions over several domain ontologies capturing concepts that crosscut several domain conceptualizations. For the purpose of this article, core ontologies are seen on par with domain ontologies. For example, the SWEET ontology or the O\&M (Observations and Measurements) ontology, both of which are discussed in Section 6 are examples of core ontologies.

${ }^{2}$ https://github.com/bfo-ontology/BFO/wiki

${ }^{3}$ https://www.w3.org/OWL/

${ }^{4}$ https://www.iso.org/standard/39175.html
} 
- GFO $[38]^{5}$ (General Formal Ontology) distinguishes processes and objects. Processes unfold in time, they have temporal parts. Objects (called presentials) have no temporal parts, and may only exist on time-boundaries. Concrete individuals exist in time or space whereas abstract individuals do not. While an endurant is an individual that exists in time, but cannot be described as having temporal parts or phases; a process, on the other hand, is extended in time. It is defined in OWL and has 243 terms.

- PROTON [86] ${ }^{6}$ (PROTo ONtology) serves as a lightweight foundational ontology organized in four modules. The top ontology module, for instance, distinguishes entity types, such as object as existing entities (agents, locations, vehicles); happening as events and situations; and abstract as abstractions that are neither objects, nor happenings. It contains about 500 classes and 150 properties ( 650 terms), providing coverage of the general concepts necessary for a wide range of tasks, including semantic annotation, indexing, and retrieval. This ontology is codified in OWL-Lite.

- SUMO $[57,62]^{7}$ (Suggested Upper Merged Ontology) is defined in the higher order logical language of SUO$\mathrm{KIF}^{8}$ It includes dozens of domains ontologies, and contains roughly 20,000 terms and 80,000 logical statements (axioms). It has an associated toolset [63], translations to languages used in theorem proving $[6,64]$ and a complete set of alignments to WordNet [58]

- UFO $[33,37]^{9}$ (Unified Foundational Ontology) has been developed with the intention of providing foundations for Conceptual Modeling. It started as an unification of the GFO and the foundational ontology of universals underlying OntoClean ${ }^{10}$ [31]. UFO is divided in three parts representing different aspects of reality: A endurants (dependent and independent objects and their types), B - perdurants (events and situations), and C social entities, with notions such as beliefs, desires, intentions, etc. UFO-A has been formalized in First-Order Modal Logics [33,36,37] (e.g., the microtheory of endurant universals contains 22 terms and 31 axioms [36]; the microtheory theory dealing with relations contains 25 terms and 20 axioms) [19]; UFO-B has been completely formalized in First-Order Logics (26 terms and 185 axioms) with a (partial) translation to $\mathcal{S R O I} \mathcal{Q}$ [4]. Taken together, the microtheory of endurants universals, the one of relations, and UFO-B amount to circa 67 terms and 236 axioms. UFO has also a partial translation to OWL termed gUFO (gentle UFO) [1], which is composed of 51 terms (class declarations) and 595 axioms.

This list summarized in Table 1 gives an idea of the variety of foundational ontologies. One can see the variety in number of entities ranging from dozens to thousands, on the other hand there is some uniformity in adoption of the OWL standard in a majority of the listed ontologies. We point out as well that most ontologies do not publish versioned releases and exact number of classes or identifiers are not available for most. We tried to provided the most up to date counts of terms or at least order of magnitude counts for the larger ones, along with the best reference available to any online repositories where the latest versions are available.

Table 1

Summary of foundational ontologies used in ontology integration, discussed in the next sections

\begin{tabular}{llll}
\hline Ontology & \multicolumn{1}{c}{ \#Entities } & \multicolumn{1}{c}{ Formats } & \multicolumn{1}{c}{ URL } \\
\hline BFO & 35 terms & OWL,CLIF & https://github.com/bfo-ontology/BFO/wiki \\
DOLCE & 100 terms/axioms (depends on the version) & OWL,KIF & http://www.loa.istc.cnr.it/ontologies/DOLCE-Lite.owl \\
Cyc & $100 \mathrm{k}$ terms and 1M axioms & CycL & http://www.cyc.com \\
GFO & 86 terms & OWL & http://www.onto-med.de/ontologies/gfo/ \\
PROTON & 650 terms & OWL-Lite & http://www.ontotext.com/proton/protontop.html \\
SUMO & 20.000 terms, 80.000 axioms & SUO-KIF & http://www.ontologyportal.org \\
UFO & 51 terms, 595 axioms (depends on the version) & FOL, SROIQ (OWL) & https://nemo-ufes.github.io/gufo/ \\
\hline
\end{tabular}

\footnotetext{
${ }^{5}$ http://www.onto-med.de/ontologies/gfo/

${ }^{6}$ http://ontotext.com/proton

${ }^{7} \mathrm{http}: / /$ www.ontologyportal.org

${ }^{8}$ https://github.com/ontologyportal/sigmakee/blob/master/suo-kif.pdf

${ }^{9}$ http://dev.nemo.inf.ufes.br/seon/UFO.html

${ }^{10} \mathrm{http}: / / \mathrm{www} . o n t o c l e a n . o r g$
} 
The list is also not exhaustive as we describe only the ontologies that are more often cited in the task of ontology matching. There are other top or foundational ontologies such as SOWA's ontology, ${ }^{11}$ YAMATO [55], GIST [89], ${ }^{12}$ KYOTO, ${ }^{13}$ PSL (Process Specification Language (PSL) [29] and BORO (Business Objects Reference Ontology) [16].

\subsection{Ontology matching}

Ontology matching refers to a process that consists of generating an alignment $\left(A^{\prime}\right)$ from a set of ontologies $\Omega$, usually a pair of ontologies $\left(\Omega=\left\{o_{1}, o_{2}\right\}\right)$.

Definition 1 (Matching process). The matching process can be seen as a function $f$ which, from a pair of ontologies $o_{1}$ and $o_{2}$ to match, an input alignment $A$, a set of parameters $p$, and a set resources $r$, returns an alignment $A^{\prime}$ between $o_{1}$ and $o_{2}$ :

$$
A^{\prime}=f\left(o_{1}, o_{2}, A, p, r\right)
$$

Each of the elements featured in this definition can have specific characteristics which influence the difficulty of the alignment task.

An alignment $\left(A\right.$ or $\left.A^{\prime}\right)$ consists of a set of correspondences $\left\{c_{1}, c_{2}, \ldots, c_{x}\right\}$ :

Definition 2 (Alignment). An alignment $A_{o_{1} \rightarrow o_{2}}$ is a set of correspondences $\left\{c_{1}, c_{2}, \ldots, c_{n}\right\} . A_{o_{1} \rightarrow o_{2}}$ is directional between a source ontology $o_{1}$ and a target ontology $o_{2}$.

A correspondence expresses a relation $r$ between ontology entities of $o_{1}$ and $o_{2}$. Here, the ontology entities are members of the correspondence:

Definition 3 (Correspondence). A correspondence $c_{i}$ is a tuple $\left(e_{1}, e_{2}, r\right) . e_{1}$ and $e_{2}$ are the members of the correspondence. They can be simple or complex expressions with entities from respectively $o_{1}$ and $o_{2}$ :

- if the correspondence is simple, both $e_{1}$ and $e_{2}$ are simple expressions.

- if the correspondence is complex, at least one of $e_{1}$ or $e_{2}$ is a complex expression, involving union, intersection, disjunction, cardinality restrictions, etc.

- $r$ is a relation, e.g., equivalence $(\equiv)$, more general $(\sqsupseteq)$, more specific $(\sqsubseteq)$, disjointedness $(\perp)$ holding between $e_{1}$ and $e_{2}$.

The correspondence $\left\langle e_{1}, e_{2}, r\right\rangle$ asserts that the relation $r$ holds between the ontology entities $e_{1}$ and $e_{2}$. One has to distinguish between more and less rigorous interpretations of relations. The equivalence relation, for example, can be interpreted as logical equivalence or more informally as a high level of similarity. Back to the example in the introduction, consider the fragment of the ontologies $e k a w^{14}$ and $c m t^{15}$ in Figs 1 and 2, respectively. The format used to represent the ontologies is described in [83]. The following correspondences can been established between these two ontologies: (i) (ekaw:Paper,cmt:Paper, $\equiv$ ) is a simple correspondence; (ii) (ekaw:AcceptedPaper, $\exists$ cmt:hasDecision.cmt:Acceptance, $\equiv)$ is a complex correspondence with constructors.

While the RDF alignment format provided in the Alignment API ${ }^{16}$ [14] is the format de facto used in the Ontology Alignment Evaluation Initiative (OAEI) campaigns for representing simple alignments, correspondences can also be represented as OWL 2 subclass, equivalence, and disjointedness axioms (with confidence values represented as axiom annotations). Alternatively, the EDOAL ${ }^{17}$ language (Expressive and Declarative Ontology Alignment Language) extends the alignment format in order to represent complex correspondences.

\footnotetext{
${ }^{11}$ http://www.jfsowa.com/ontology/toplevel.htm

$12 \mathrm{https}: / / \mathrm{www}$. semanticarts.com/gist/

$13 \mathrm{http} / / /$ kyoto-project.eu/xmlgroup.iit.cnr.it/kyoto/index.html

${ }^{14}$ http://oaei.ontologymatching.org/2019/conference/data/ekaw.owl

15 http://oaei.ontologymatching.org/2019/conference/data/cmt.owl

16 http://alignapi.gforge.inria.fr/format.html

${ }^{17}$ http://alignapi.gforge.inria.fr/edoal.html
} 


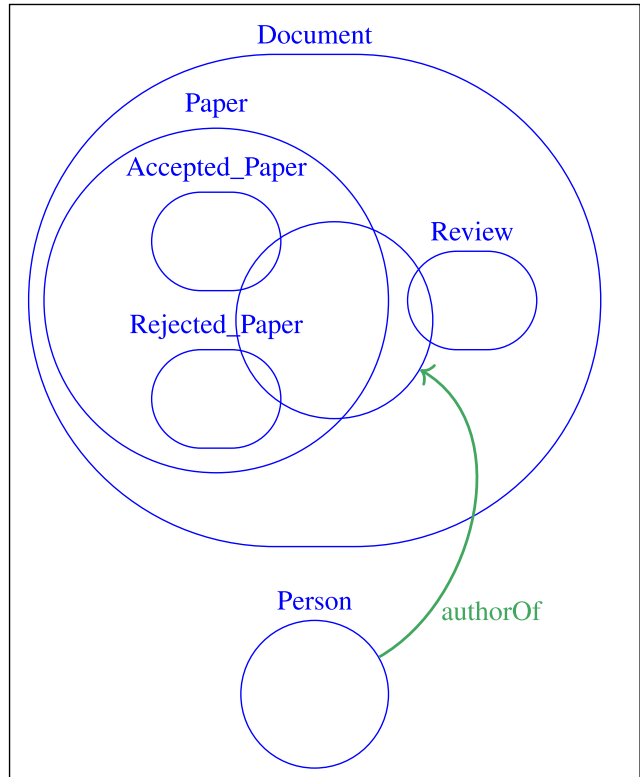

Fig. 1. Fragment of ekaw ontology (adapted from [87]).

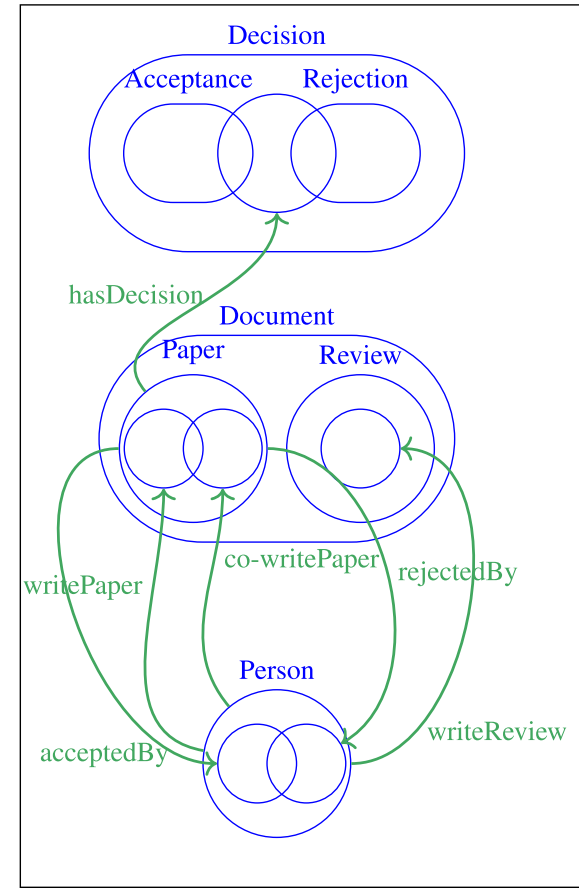

Fig. 2. Fragment of cmt ontology (adapted from [87]).

In the following, we discuss the use of foundational ontologies in different matching tasks: (i) matching of foundational ontologies; (ii) matching of foundational ontologies to lexicons; (iii) matching domain ontologies with the help of foundational ontologies; and (iv) matching foundational ontologies to domain ontologies. 


\section{Matching foundational ontologies}

As stated in [42], while the purpose of a foundational ontology is to address interoperability among ontologies, the development of different foundational ontologies re-introduces the interoperability problem. As briefly discussed in the previous section, these ontologies have been developed directed at different classes of applications, as well as relying on different theoretical assumptions.

Early work addressed this problem $[27,79,85]$ from different perspectives on the alignments. While [27] compared specific treatments of fundamental issues (as significant discrepancies related to universals and particulars, qualities, constitution and spatio-temporality) and how similar notions apply differently in BFO and DOLCE, [79] compared the primitive relations (dependence, quality, and constitution) between these ontologies. In [85], the alignment between BFO and DOLCE was established in order to conciliate their respective realistic and cognitive points of view and to integrate medical data. While $100 \%$ of BFO categories were aligned to DOLCE, $81 \%$ of DOLCE categories were aligned to BFO.

More recently, [90] compares BORO and UFO ontologies according to the their metaphysical choices that define their structure and composition. Instead of comparing terms in both ontologies, the authors compare how the two approaches address issues such as identity and dynamic classification, the treatment of relationships (i.e., instances of relational properties), as well as the relation between existence and time in the two approaches. The radical difference between these two ontologies, hence, reflect deeper differences in ways of conceiving reality.

Other studies addressed other foundational ontologies. In [42], alignments between BFO, DOLCE and GFO have been established with automatic matching tools and manually, with substantially fewer alignments found by the matching tools. The alignments in the context of the whole ontology revealed a considerable number of logical inconsistencies. This work has been extended in [73] in two ways: considering matching systems participating in OAEI 2018, and a new pair of aligned foundational ontologies (SUMO and DOLCE). The alignments in [42] and [59] served as a reference alignment to automatically evaluate the matchers. Examples of reference correspondences include: (bfo:Occurrent,dolce-lite:perdurant, $\equiv$ ) and (dolce-lite:artifact,sumo:Device, $\sqsupseteq$ ).

Overall, the results found are in line with what has been reported when evaluating the behaviour of matchers in the task of matching domain and foundational ontologies, which would also require identification of subsumption relations [74]. Current tools fail on correctly capturing the semantics behind the ontological concepts, which requires deeper contextualization of the concepts on the basis of their hierarchy and axioms. Addressing the identification of subsumption relations, the approach in [40] relies on extracting hypernym relations from ontology annotations for establishing such kind of correspondences. Results on exploiting lexico-syntactic patterns and definitions layout on DOLCE and SUMO were evaluated on a manually generated subsumption reference.

From another perspective, the core characterization of mereotopology (a theory of physical parts) of SUMO and DOLCE has been studied in [56], relating their axiomatizations via ontology alignments. This included corrections and additions of axioms to the analyzed theories which eliminate unintended models and characterize missing ones. Finding alignments between DOLCE and SUMO was also addressed in [59], where the SmartDOLCE and SmartSUMO ontologies have been developed on the basis of DOLCE and SUMO. The alignment of the just the taxonomic statements from SUMO to DOLCE involved extracting the upper-level of SUMO and the non-trivial task of aligning the remaining concepts to appropriate DOLCE categories.

Aligning foundational ontologies reveals also the problem of matching their different versions. In [78], a method for tracking, explaining and measuring changes between successive versions of BFO1.0, BFO1.1, and BFO2.0 was applied. The aim was to provide a more comprehensive analysis of the changes with respect to the BFOConvert tool $^{18}$ which provides an alignment between previous BFO versions, as this resource is limited to allow for a full understanding of the impact of the changes.

Formalizeations [10] within the Common Logic Ontology Repository (COLORE), were used in the specification of alignments between upper ontologies in [28]. These alignments serve for the verification of foundational ontologies. Similarly, [11] shows how to apply techniques for ontology verification to link interpretations among ontologies.

\footnotetext{
${ }^{18} \mathrm{http}: / /$ ontobull.hegroup.org/bfoconvert (viewed on 25/03/2019).
} 
Table 2

Summary of matchings of foundational ontologies ( ${ }^{*}$ http://www.thezfiles.co.za/ROMULUS/ontologyAlignment.html)

\begin{tabular}{llll}
\hline & \multicolumn{1}{c}{ Matching foundational ontologies } \\
\hline Work & \multicolumn{1}{c}{ Ontologies } & \multicolumn{1}{c}{ Approach } & \multicolumn{1}{c}{ Available alignment } \\
\hline$[27]$ & BFO 1.0, DOLCE & Manual comparison & - \\
{$[79]$} & BFO 1.0, DOLCE & Manual comparison & Set of triples \\
{$[85]$} & BFO 1.0, DOLCE & Manual alignment & List at Romulus* \\
{$[42]$} & BFO 1.1, DOLCE-Lite, GFO & Manual, matching tools & - \\
{$[78]$} & BFO 1.0, BFO 1.1,BFO 2.0 & Semi-automatic (change-tracking) & - \\
{$[59]$} & SUMO, DOLCE & Manual alignment & FOL alignments \\
{$[56]$} & SUMO, DOLCE-CORE & Manual alignment & Alignment API format \\
{$[73]$} & BFO, GFO, DOLCE-Lite, SUMO & Matching tools & Alignment API format \\
{$[40]$} & DOLCE-Lite, SUMO & Hypernym relation extraction &
\end{tabular}

Table 2 summarizes work on matching of foundational ontologies. They are mostly manual efforts, with a few of the resulting alignments made available.

\section{Matching foundational ontologies to lexicons}

Several efforts in equipping lexical resources with foundational ontologies have been made in order to associate a formal semantics to their lexical layer. As stated in [20,21], while WordNet has been used in numerous work as an ontology, where the hyponym relations between word senses are interpreted as subsumptions relation between concepts, it is only serviceable as an ontology if some of its links are interpreted according to a formal semantics that tell us something about the world and not just about language.

For example, WordNet has the sense of "chair" as a hyponym of "seat" but only an English gloss meant for humans to read for each, and no logical semantics that defines the hyponym link, with the result that hyponyms are often incorrectly treated by users as being logically transitive. An ontology would, at a minimum, define the axiom of transitivity, and state that it holds on the hyponym relation. Most ontologies have little more than class/subclass relations however. One might also want to state that a chair is a artifact made by humans with the intent for use in supporting a seated humans. Some upper ontologies have axioms that define their terms, and some large taxonomies have terms for specific things like chairs, but very few have axiomatized large number of detailed objects, processes and relationships. This poses a problem for matching algorithms if there aren't both detailed formalizations that can be used to objectively determine a correct match and a large inventory so that matches are not to trivially general terms like object or event.

A number of researchers have investigated different ontological problems in treating WordNet as an ontology (e.g., confusion between concepts and individuals, constraints violations, heterogeneous levels of generality, etc.) [20] and provided the WordNet taxonomy with more rigorous semantics. First the WordNet taxonomy was reorganized to meet the OntoClean [31] methodology requirements, and the resulting upper level nouns were then mapped to DOLCE classes representing their highest level categories. This alignment is concentrated on the noun database, since most particulars in DOLCE describe categories whose members are denoted by nouns. The result is the OntoWordNet resource expressing alignments between WordNet 1.6 version and DOLCE Lite Plus. An extension is presented in [24] in order to extract association relations from WordNet, and to interpret those associations in terms of a set of conceptual relations in DOLCE.

Later, this alignment has been updated [25] with a revision of the manual alignments and different versions of DOLCE and WordNet, WordNet 3.0 and (DOLCE UltraLitePlus), which is a simplified version of DOLCE Lite Plus, intended to make classes and properties names more intuitive and express axiomatizations in a simpler way, among other features.

While these works focused mostly on WordNet noun synsets, [81] extended the previous alignments by aligning verbs according to their links to nouns denoting perdurants, transferring to the verb the DOLCE class assigned to the 
Table 3

Summary of matching with lexicons (*http://www.ontologydesignpatterns.org/ont/wn/)

\begin{tabular}{llll}
\hline & \multicolumn{2}{c}{ Matching foundational ontologies to lexicons } \\
\hline Work & \multicolumn{1}{c}{ Ontologies } & \multicolumn{1}{c}{ Approach } & \multicolumn{1}{c}{ Available alignment } \\
\hline$[22,24]$ & DOLCE-LitePlus,DOLCE-UltraLite/WordNet1.6 & Semi-automatic (NLP, disamb., A-links) & OWL version* \\
{$[25]$} & DOLCEPlusDnS Ultra Lite/WordNet3.0 & Semi-automatically (transitive closure) \\
{$[81]$} & DOLCE-LitePlus/WordNet3.0 (verbs) & Semi-automatic (annotation tool, links) & RDF dataset \\
{$[77]$} & BFO2.0/WordNet3.0 & Semi-automatic (matching rules) & - \\
{$[67]$} & Cyc/WordNet1.6 & Semi-automatic (interactive tool, rules) & Tanual \\
{$[58]$} & SUMO/WordNet1.6/3.0 & Semi-automatic & SUMO axioms \\
{$[17]$} & SUMO, YAGO, WordNet, Wikipedia & Automatic (SemanticMapper) & - \\
{$[45]$} & UF0/WordNet3.0 & &
\end{tabular}

noun that best represents that verb's occurrence. They argue that many NLP applications need to deal with events, actions, states, and other temporal entities that are usually represented by verbs.

The alignment of WordNet to other foundational ontologies has been also addressed. In [77], a semi-automatic method for aligning WordNet 3.0 and BFO2.0 is described. It adopts previous alignments between WordNet and the KYOTO ontology, whose top layer is based on DOLCE. The method involves manually creating a set of alignments between the ontologies and implementing a set of matching rules.

In [67], the authors report the matching and integration of several background resources and ontologies of varying complexity to the Cyc knowledge base. These resources and ontologies included large pharmaceutical and medical thesauri and large portions of WordNet. For this task, ontologists have been trained with domain experts and interactive clarification dialog-based tools were developed to enable experts to directly match/integrate their ontologies. In [58], SUMO was originally mapped manually to WordNet 1.6 and then manually updated to $3.0 .{ }^{19}$ It is the only manual alignment of an ontology to every word sense in WordNet.

SUMO and WordNet were used in a semi-automated process to match the millions of terms in the YAGO $^{20}$ taxonomy and create a single large ontology and Factbase [17].

Finally, in [45], WordNet has been extended by applying the notion of semantic types in order to establish matching rules between the noun synsets of WordNet and the top-level constructs of the UFO ontology. The proposed rules were validated through an experiment with approximately 5,200 sample correspondences and average accuracy of $93 \%$.

Table 3 summarizes the works presented in this section. There are some available alignments, approaches are mostly semi-automatic, with one case of manual alignment and one case of automatic alignment. WordNet is the lexical resource that is considered in all work listed and two different versions are involved in the alignments (1.6 and 3.0).

\section{Matching domain via foundational ontologies}

Foundational ontologies provide a reference for rigorous comparisons of different ontological approaches, and a framework for analysing, harmonizing, matching and integrating existing domain ontologies [59]. In domain ontology matching, in particular, they act as semantic bridges to help the task. For instance, reducing the matching space to the entities under a same category e.g., avoiding matching dolce:endurants to dolce:perdurants.

Despite the potential gain of exploiting foundational ontologies in domain ontology matching, few works have addressed this alternative, possibility due to the still lack of systematic alignments between domain and foundational ontologies. This gain has been quantitatively measured in [51], where a set of algorithms exploiting such semantic bridges are applied. The circumstances of cases where foundational ontologies improve domain ontology matching,

\footnotetext{
${ }^{19}$ https://github.com/ontologyportal/sumo/tree/master/WordNetMappings

${ }^{20} \mathrm{http}: / /$ yago.r2.enst.fr
} 
Table 4

Summary of matching via foundational ontologias

\begin{tabular}{llll}
\hline & & \multicolumn{1}{c}{ Matching domain ontologies via foundational ontologies } \\
\hline Work & \multicolumn{1}{c}{ Ontologies } & \multicolumn{1}{c}{ Approach } & \multicolumn{1}{c}{ Available alignment } \\
\hline$[48]$ & SUMO, Cyc/SENSUS & Semi-automatic (LOM matcher) & - \\
{$[51]$} & SUMO-OWL, OpenCyc, DOLCE/17 ont. (agent, bibtex, etc.) & Automatic (structural matching) & - \\
{$[82]$} & BFO/GO, INOH Event & Automatic (FOAM+OBOAEA) & - \\
{$[60]$} & UFO/Conference & Manual pattern analysis & - \\
\hline
\end{tabular}

with respect to approaches ignoring them, were then studied. The experiments were conducted with SUMO-OWL (a restricted version of SUMO), OpenCyc and DOLCE and demonstrate that overall the alignment via upper ontologies impacts in F-measure positively. Additionally, in [60] a set of alignment patterns based on OntoUML (a conceptual modeling language based on UFO) are applied to a set of alignments generated by matching systems. An analysis of the impact of patterns to avoid common errors was presented.

The semi-automatic LOM matcher [48] combines WordNet synset matching (checking terms from the ontologies to be matched sharing common synsets) and type matching. This strategy employs SUMO to determine the ontological category of each word constituent for matching using the alignments from WordNet synsets to SUMO. LOM takes the source terms and collects the set of SUMO terms that their synsets map to, and then compares the SUMO term sets to their counterpart for each term in the target ontology.

From a manually established alignment between biomedical ontologies and BFO, in [82], a matching approach relies on filtering out correspondences at domain level that relate two different kinds of ontology entities. The matching approach is based on a set of similarity measures and the use of foundational ontology as a parameter for better understanding the conceptual nature of terms within the similarity calculation step. Besides the reported improvement in the results obtained, the introduction of foundational ontologies in the alignment process increased the influence of semantic factors in this task, further expanding the universe of information to be explored during the alignment.

Table 4 summarizes the use of foundational ontologies as an aid to the of matching domain ontologies. Here the automatic approaches are adopted more frequently. Alignments, however, were not found to be available.

\section{Matching domain to foundational ontologies}

Methodologies for constructing ontologies should not neglect the use of foundational ontologies and may better address it in a top-down approach [2,41]. As one example, existing methodologies including OntoUML, allow for creating a domain or core ontology compliant to UFO [37].

While matching foundational ontologies is mostly manually done, with more automation in matching domain ontologies via foundational ones, in this section, both approaches have been performed.

\subsection{Manual alignment}

Many approaches for mapping rely on a manual alignment process. In [8], DOLCE was used to integrate two geoscience knowledge representations, the GeoSciML schema and the SWEET ontology, in order to facilitate crossdomain data integration. The aim was to produce a unified ontology in which the GeoSciML and SWEET representations are aligned to DOLCE and to each other. In that perspective, DOLCE works as a semantic bridge and this approach fits in the category of domain matching with foundational ontologies. The alignments have been manually established and representation incompatibility issues have been discussed so far. Similarly, in [66], manual alignments were established between the O\&M (Observations and Measurements) ontology and DOLCE, in order to restrict the interpretations of entities in the O\&M model and to make explicit the relations between their categories.

DOLCE has been manually aligned to the domain ontology describing services (OWL-S) in [52], in order to address its conceptual ambiguity, poor axiomatization, loose design and narrow scope. They have also developed a 
Table 5

Fragment of the spreadsheet for the manual alignment between $\mathrm{cm}$ t ontology in Fig. 2 and SUMO, via WordNet

\begin{tabular}{lll}
\hline Domain concept & \multicolumn{1}{c}{ WordNet synsets (S) } & \multicolumn{1}{c}{ SUMO concept } \\
\hline cmt\#Conference & S1: conference group discussion & 1: Communication+ \\
& S2: league conference & 2: SportsLeague+ \\
& S3: conference & 3: FormalMeeting \\
cmt\#Decision & S1: decision determination conclusion & 1: Learning+ \\
& S2: decision determination conclusion & 2: Deciding+ \\
& S3: decisiveness decision & 3: TraitAttribute+ \\
& S4: decision & 4: ConstantQuantity+ \\
& S5: decision & 5: ConstantQuantity+
\end{tabular}

core ontology of services to serve as middle level between the foundational and OWL-S, and can be reused to align other Web Service description languages.

In [13], several schemata of FactForge, which enables SPARQL queries over a LOD cloud, have been aligned to the foundational ontology PROTON in order to provide a unified way to access to the data. The alignments were created by knowledge engineers through a manual process. Equivalence e.g., (geonames:Country,proton:Country,

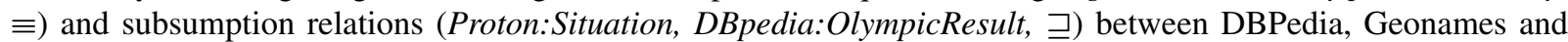
Freebase concepts and PROTON classes have been established. In other similar work, [12] includes an open-source extract of the OpenCyc knowledge base (UMBEL).

As stated in Section 5, manual alignments have also been established between biomedical ontologies and BFO, in [82]. In this line, [9] analysed the "compatibility" between an ontology of the biomedical domain (UMLS) and the Cyc Ontology, by manually aligning UMLS to Cyc.

In [72], ontologies from the OAEI Conference track have been manually aligned to SUMO. As a complete manual alignment between SUMO and WordNet is available, such alignments have been used as bridges to facilitate the matching task. Four annotators have been worked on the alignments. Table 5 shows a fragment of the spreadsheet used for the annotators to align the domain concepts to the SUMO concepts.

During the process of alignment, several difficulties arose for interpreting the real meaning that the concept represents in the domain ontology. For instance, the concepts $\mathrm{cmt}$ : Bid and cmt : Preference had no description clarifying theiruse, and no sub- or super-concepts that could be used to clarify their meaning. In addition, some concepts represented in the ontology present other kind of problems such as doubts regarding ontology elements' adequacy, for example, the concept edas : ReviewRating, for which, according to the discussion raised by the evaluators, a rating could be a relationship between a thing, an agent and a rating value, rather than a class or individual as it would appear.

In contrast, one can examine a SUMO definition of a term such as sumo: FormalMeeting and see that it is necessarily a sumo: Meeting which is not a sumo: SocialParty, that it must be temporally preceded by a sumo:Planning which has the result of creating the meeting, as well as constraints that other events like a sumo: Resolution, which to be considered as such, may only occur at a sumo: FormalMeeting. It provides information something like the level of detail in a modern dictionary, but with the definitions expressed in logic, rather than human language, so that a machine can perform computation (and consistency checking) with those definitions. The cases described above consist of ontological representation problems commonly present in lightweight ontologies, and hinder the reuse and reliability of the represented knowledge. In addition, they highlight the importance of advancing in research that uses foundational ontologies to give more formalization to domain ontologies.

In [61], existing alignments between DBPedia ontology and DOLCE-Zero ${ }^{21}[21,23]$, a subset of the modules of the formal ontology, are used to identify inconsistent statements (systematic errors or anti-patterns) in DBPedia. DOLCE-Zero simplifies some of the distinctions in DUL, which has been created to optimize the alignment of WordNet used by the Tipalo method for automatic typing of Wikipedia resources [25]. Systematic errors are sets of

${ }^{21}$ http://www.ontologydesignpatterns.org/ont/dul/d0.owl 
individual errors following a similar pattern and having a common root cause (e.g., a wrong correspondence). The alignment has been defined by a DOLCE-Zero designer. By using these alignments and by combining reasoning and clustering of the reasoning results, errors affecting statements can be identified at a minimal human workload.

\subsection{Automatic and semi-automatic alignment}

While the previous proposals mainly generate manual alignments, BLOOMS + [39] is an early work on automatising the process. It has been used to automatically align PROTON to LOD datasets using as gold standard the alignments provided in [13]. BLOOMS + first uses Wikipedia to construct a set of category hierarchy trees for each class in the source and target ontologies. It then determines which classes to align using 1) similarity between classes based on their category hierarchy trees; and 2) contextual similarity between these classes to support (or reject) an alignment. BLOOMS + significantly outperformed existing matchers in the task.

In [69] the authors have proposed an automatic approach for matching domain and foundational ontologies that exploits existing alignments between WordNet and foundational ontologies. The matching process is divided in two main steps. The first step identifies the correct synset to a concept and the second one identifies the correspondence of a domain concept to a foundational concept. The approach has been evaluated using DOLCE and domain ontologies from the OAEI conference data set, ${ }^{22}$ with the help of the alignments provided in [24,58]. This work has been further extended in [70], where two similarity measures for synset disambiguation have been adopted: (1) an adaptation of the Lesk [47] measure and (2) word embeddings [53] similarity. The evaluation has been also extended including DOLCE and SUMO ontologies and their alignments to WordNet and three domain ontologies $\left(\mathrm{SSN},{ }^{23} \mathrm{CORA},{ }^{24}\right.$ and OAEI Conference). SSN (W3C Semantic Sensor Network Ontology) describes sensors, devices, observations, measurements and other terms, enabling reasoning of individual sensors and the connection of them. A recent version of SSN includes a lightweight core ontology called SOSA (Sensor, Observation, Sample, and Actuators). SSN is aligned to SOSA and both ontologies are aligned to DOLCE Ultralite (DUL). SSN is composed of 18 first level concepts, from those, 8 concepts are aligned to the top ontology DUL. CORA (IEEE Core Ontology for Robotic and Automation) [65] is an effort of the IEEE Ontologies for Robotics and Automation Working group (ORA). It specifies the main concepts, relations, and axioms of robotics and automation domains. CORA is aligned to the SUMO top-level ontology. CORA is composed of 34 first level concepts, from which 29 of them are aligned to SUMO.

In [49] WordNet was used as background knowledge, and their matching approach combines concept definition enrichment, disambiguation and filtering of candidate correspondences with inconsistency detection. The approach has been used for matching DOLCE+DnS Ultralite and a domain ontolology describing mobile services.

Automatic foundational distinctions of LOD entities (class vs. instance or physical vs. non-physical objects) is done in [3] with two strategies: an (unsupervised) alignment approach and a (supervised) machine learning approach. The alignment approach, in particular, relies on the linking structure of alignments between DBpedia, DOLCE, and lexical linked data, using resources such as BabelNet, YAGO and OntoWordNet. For instance, they use the paths of alignments and taxonomic relations in these resources and automated inferences to classify whether a DBpedia entity is a physical object or not.

Table 6 summarizes work in alignments between domain and foundational ontologies. Several efforts have been made to align DOLCE to domain ontologies, SUMO and PROTON are also considered in more than one work. These projects use different domain ontologies, and results are unavailable is most of the cases, therefore a detailed third-party comparison of approaches is yet not possible.

\section{Discussion}

The following sections discuss a series of issues regarding matching of foundational ontologies: the complexity of the task, the automation of systems with capabilities to include such alignments, the lack of evaluation data sets,

\footnotetext{
${ }^{22}$ http://oaei.ontologymatching.org/2017/conference/index.html

${ }^{23} \mathrm{https}: / / \mathrm{www} . \mathrm{w} 3$. org/TR/vocab-ssn/

${ }^{24}$ IEEE Standard Ontologies for Robotics and Automation,” in IEEE Std 1872-2015, vol., no., pp.1-60, 10 April 2015.
} 
Table 6

Summary of matching with domain ontologies and cross-domain ontologies ( ${ }^{*}$ https://github.com/danielasch/top-match)

\begin{tabular}{llll}
\hline \multicolumn{1}{c}{ Matching domain ontologies to foundational ontologies } \\
\hline Work & \multicolumn{1}{c}{ Ontologies } & \multicolumn{1}{c}{ Approach } & Manual \\
\hline$[52]$ & DOLCE/OWL-S & Manual & Unailable alignment \\
{$[8]$} & DOLCE-LitePlus/GeoSciML2.0, SWEET1.1 & Automatic (lexical+reasoning) & - \\
{$[49]$} & DOLCE Ultralite/Mobile services ontology & Automatic (indirect matching) & - \\
{$[69]$} & DOLCE-LitePlus/OAEI Conference & Automatic (indirect+embeddings) & Alignment API format [14] \\
{$[70]$} & DOLCE-LitePlus, DOLCE Ultralite, SUM0/Conf, SSN, CORA & - \\
{$[3]$} & DOLCE-LitePlus/DBPedia & Automatic (machine learning) & - \\
{$[13]$} & PROTON/DBPedia, Freebase, Geonames & Manual & - \\
{$[39]$} & PROTON/DBPedia, Freebase, Geonames & Automatic (BLOOMS+) & - \\
{$[12]$} & PROTON, OpenCyc(UMBEL)/Freebase, Geonames & & - \\
{$[82]$} & BFO/GO, INOH Event & Manual & - \\
{$[72]$} & SUMO/OAEI Conference & Manual (via WordNet) & http://purl.org/ieee1872-owl \\
{$[65]$} & SUMO/CORA Robotics & Manual &
\end{tabular}

the evolution of different versions and the problems that poses, the desired variety and lack of expressiveness in the alignments, and finally, multilingualism.

\subsection{Complexity of the task}

The problem of matching ontologies gets more complex when involving foundational ontologies, that explains why there is less development regarding automatic solutions [44,70]. It requires the deep identification of the semantic context, the identification of subsumption relations, and consistentency with the formalization. In fact, subsumption and other relations are often neglected by most state-of-the-art matchers.

As seen in the previous sections, most approaches still rely on manually or semi-automatically established alignments. This task is far from being trivial, even when done manually. This has been recently corroborated in [84], where manually classifying domain entities under foundational ontology classes is reported to be very difficult to do correctly. Manual ontology matching is also an expensive task that may introduce a bias as it represents a point of view expressing the interpretation of the concepts influenced by the background of the expert. As knowledge on foundational ontologies is specialized, it is important that such evaluation considers an overview of different experts in this area. Moreover, while manual alignment on a small set of concepts is feasible, bigger data sets would require considerable effort. The findings in [84] also point out the need for improving the methodological process of manual integration of domain and foundational ontologies, in accordance with what has been stated in [41].

\subsection{Automation}

While more automation is an obvious requirement in the field, the poor performance of solutions addressing automatically matching different foundational ontologies or with domain ontologies have demonstrated the difficulty of the task, as reported in experiments evaluating current matching tools [42,71]. Current tools fail on correctly capturing the semantics behind concepts (even when such semantics are present), which requires deeper contextualization on the basis of hierarchies and axioms. In that sense, further context and documentation is required, in particular for domain ontologies, to help identifying the right semantics (e.g, the ontologies from the largely used OAEI Conference dataset have a very poor lexical layer and limited or non-existent axiomatized semantics).

Furthermore, while diverse (domain) ontology matching approaches rely on external background knowledge, (BabelNet, ${ }^{25}$ WordNet, ${ }^{26}$ UMLS, ${ }^{27}$ etc.), the coverage of foundational ontologies in these resources is still low. More

\footnotetext{
${ }^{25}$ https://babelnet.org

${ }^{26} \mathrm{https}: / /$ wordnet.princeton.edu

${ }^{27}$ https://uts.nlm.nih.gov/home.html
} 
recently, the resource Framester, ${ }^{28}$ exposed as a knowledge graph, addresses this aspect as a hub between several resources such as VerbNet, ${ }^{29}$ BabelNet, DBpedia, ${ }^{30}$ and YAGO. ${ }^{31}$ Hence, matchers need to be improved to include more abstract and philosophical semantic relations and semiotic matching, to take advantage of structural features of the ontologies and axioms in order to better compare their formal definitions, and also of background knowledge from external resources, targeting subsumption and other relations. These have to be combined with logical reasoning techniques for guarantee the consistency of the generated alignments. The current approaches have to be thus revised to better deal with the specificities of matching with foundational ontologies. While automatic approaches have been mostly manually evaluated, with few exceptions $[13,70]$, systematically evaluations of matching systems have been so far dedicated to domain ontologies.

\subsection{Other relations than equivalence}

Besides the points raised above, the task requires the identification of other relations than equivalences, such as subsumption and meronymy. The latter is largely neglected by current matchers. In particular, the main problem of matching foundational and domain ontologies is that, most matchers typically rely on string-based techniques as an initial estimate of the likelihood that two elements refer to the same real world phenomenon, hence the found correspondences represent equivalences with concepts that are equally or similarly written. However, in many cases, this correspondence is not the case [71]. In fact, when having different levels of abstraction it might be that the matching process is capable of identifying subsumption correspondences rather than equivalence, since the foundational ontologies have concepts at a higher level.

\subsection{Evaluation}

Despite the variety of tasks in the OAEI campaigns, ${ }^{32}$ evaluations involving foundational ontologies have not been addressed. Producing comprehensive evaluation data sets on which matching solutions can be evaluated would foster the development of approaches involving foundational ontologies and support a next generation of semantic matching approaches. With that respect, few of the established alignments generated by the approaches have being publicly made available. Furthermore, very few of them adopted a format that can be processed by automatic tools. Only [69] adopts the Alignment API Format, the standard de facto adopted in the OAEI campaigns.

\subsection{Versioning}

Another aspect refers to the evolution or the consistency of alignments with respect to the evolution or the different variants of the ontologies. For example, DOLCE and its different variants have been used in diverse proposals, as many efforts have been dedicated to the development of this ontology. DOLCE has been exposed with reduced axiomatization and extensions with generic or domain plugins, such as for DOLCE-Lite [24], DOLCE Lite Plus ${ }^{33}$ or still DOLCE+DnS Ultralite. ${ }^{34}$ Besides their substantial differences in the hierarchical organization and expressiveness, these versions are mostly compatible, what is not the case for other ontologies. For instance, BFO 2.0 represents major updates to BFO not strictly backwards compatible with BFO 1.1 and a manual alignment was required to express their incompatibilities. UFO is also currently being extended by incorporating a new theory of types (including higher-order types), as well as a fuller theory of relationships and events [37]. Despite being, to a large extent, backwards compatible with the original ontology, these are important changes of UFO 2.0.

Another issue is related to the evolution of the resources aligned to the ontologies. As stated in [61], conflicts may arise between an alignment defined on a version, and a newer version. The alignment provided for an older version

\footnotetext{
${ }^{28}$ https://lipn.univ-paris13.fr/framester/

${ }^{29} \mathrm{https}: / /$ verbs.colorado.edu/verbnet/

30 https://wiki.dbpedia.org

${ }^{31} \mathrm{http}: / /$ yago.r2.enst.fr

${ }^{32}$ http://oaei.ontologymatching.org/2020/

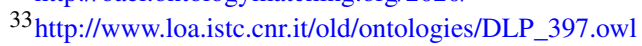

${ }^{34} \mathrm{http}: / /$ www.ontologydesignpatterns.org/ont/dul/DUL.owl
} 
may become incoherent in case of a non-conservative change of the ontology in the newer version. It is the case in the alignments between DOLCE and the different versions of DBPedia. Taking the example presented by the authors, for instance, dbo:team used to hold between career stations (professional situations of e.g. an athlete) and teams in DBpedia 3.9 ontology, but in DBpedia 2014 it holds between agents and sports teams. Since dul:Situation (aligning dbo:CareerStation), and dul:Person (aligning dbo:Athlete are disjoint, inconsistencies are detected. In particular, in particular where a) the DBpedia2014 ontology has used DBpedia 3.9 alignments, but the basic ontology had changed; and b) some properties are applied ambiguously, which should lead either to a change of the alignment, or of the DBpedia data or ontology.

Evolving alignments to cope with the different versions of the ontologies is still an open challenge.

\subsection{Expressiveness}

Most alignments generated in the research we have surveyed were limited to linking of a single entity of a source ontology to a single entity of a target ontology. The links lack expressiveness to a large extent. In order to better express the relationships between entities from different ontologies, they require rather full fledged axioms, as pointed out in [13,67]. In the example from [13], a complex correspondence states that the professions are modeled as instances of the class Profession in PROTON, and the single entity of DBPedia is matched to an expression in PROTON which restricts the property hasProfession to the value of the profession of interest. However, generating complex correspondences is still an open challenge in the ontology matching field in general.

The most significant issue in ontology matching is that most ontologies lack definitions of terms in logic, comparable to the completeness of natural language definitions in dictionaries. Most of the intended semantics of terms are left to the intuition of humans reading their names. Until richer definitions become the norm, ontology matching, whether manual or automatic, will remain difficult to conduct or evaluate.

\subsection{Multilingualism}

Very few foundational ontologies are equipped with lexical layers in languages other than English (e.g., BFO has been enriched with a lexical annotation in Portuguese, SUMO is the exception and is matched to the 26 languages in Open Multilingual WordNet [7]). However, with the increasing amount of multilingual data on the Web and the consequent development of ontologies in different languages, foundational ontologies should also be equipped with richer multilingual annotations in order to facilitate the multilingual and cross-lingual ontology matching tasks.

\section{Final remarks}

Ontology matching has reached some maturity in terms of matching domain ontologies. There is however room for further developments in the adoption of foundational ontologies in the task. Systematically enriching domain ontologies with foundational ones would also promote their use as semantic bridges in the task of matching domain ontologies. One of the difficulties however is the need of specialised knowledge as injecting foundational ontologies in ontology matching, in general, requires deeply understanding the foundational concepts and its relations. Another issue concerns the lack of formal definitions associated to lexicons (comments and labels) helping to understand the precise semantics of each concept. These are among the main brakes to automatising together with the points discussed above.

This paper has provided an overview of the adoption and exploration of foundational ontologies in the task of ontology matching, on different perspectives: work attempting to compare and match foundational ontologies, natural language definitions vs logical statement issues addressed by linking lexicons and foundational ontologies, the role of foundational ontologies as bridges for linking domain ontologies; and equipping existing domain ontologies with foundational distinctions. We have pointed out the limitations to be addressed in order to bring the clarity of semantics of foundational ontologies in ontologies in general.

In a broader scope, semantic web in general and its materialisation with the linked open data initiative still lack such ontological distinction, as recently stated in $[3,88]$. This has been further corroborated in [5], where it is stated 
that in the semantic web, there is an increasingly need for serious engagement with ontology, understood as a general theory of the types of entities and relations making up their respective domains of inquiry. However, there is still little interaction between the communities, despite the fact that they share common ambitions in terms of knowledge understanding. This goes beyond the matching task as discussed in this paper in the sense that is has to take into account the data being described by the ontologies.

\section{Acknowledgement}

Renata Vieira is funded by the Portuguese Foundation for Science and Technology, project UIDB-00057-2020.

\section{References}

[1] J.P.A. Almeida, G. Guizzardi, T.P. Sales and R.A. Falbo, gUFO: A lightweight implementation of the Unified Foundational Ontology (UFO), 2019.

[2] R. Arp, B. Smith and A. Spear, Building Ontologies with Basic Formal Ontology, MIT Press, 2015.

[3] L. Asprino, V. Basile, P. Ciancarini and V. Presutti, Empirical analysis of foundational distinctions in linked open data, in: Proceedings of the Twenty-Seventh International Joint Conference on Artificial Intelligence, IJCAI 2018, Stockholm, Sweden, July 13-19, 2018, J. Lang, ed., ijcai.org, 2018, pp. 3962-3969. doi:10.24963/ijcai.2018/551.

[4] A.B. Benevides, J. Bourguet, G. Guizzardi, R. Peñaloza and J.P.A. Almeida, Representing a reference foundational ontology of events in SROIQ, Applied Ontology 14(3) (2019), 293-334. doi:10.3233/AO-190214.

[5] M. Bennett and K. Baclawski, The role of ontologies in linked data, big data and semantic web applications, Applied Ontology 12(3-4) (2017), 189-194. doi:10.3233/AO-170185.

[6] C. Benzmüller and A. Pease, Progress in automating higher-order ontology reasoning, in: Proceedings of the 2nd Workshop on Practical Aspects of Automated Reasoning, PAAR-2010, Edinburgh, Scotland, UK, July 14, 2010, R.A. Schmidt, S. Schulz and B. Konev, eds, EPiC Series in Computing, Vol. 9, EasyChair, 2010, pp. 22-32. doi:10.29007/dtnz.

[7] F. Bond, C. Fellbaum, S. Hsieh, C. Huang, A. Pease and P. Vossen, A multilingual lexico-semantic database and ontology, in: Towards the Multilingual Semantic Web, Principles, Methods and Applications, P. Buitelaar and P. Cimiano, eds, Springer, 2014, pp. 243-258. doi:10. 1007/978-3-662-43585-4_15.

[8] B. Brodaric and F. Probst, DOLCE ROCKS: Integrating geoscience ontologies with DOLCE, in: Semantic Scientific Knowledge Integration, Papers from the 2008 AAAI Spring Symposium, Technical Report SS-08-05, Stanford, California, USA, March 26-28, 2008, AAAI, 2008, pp. 3-8.

[9] A. Burgun and O. Bodenreider, Mapping the UMLS semantic network into general ontologies, in: AMIA 2001, American Medical Informatics Association Annual Symposium, Washington, DC, USA, November 3-7, 2001, AMIA, 2001, pp. 1-5.

[10] C. Chui and M. Grüninger, Mathematical foundations for participation ontologies, in: Formal Ontology in Information Systems - Proceedings of the Eighth International Conference, FOIS 2014, Rio de Janeiro, Brazil, September, 22-25, 2014, P. Garbacz and O. Kutz, eds, Frontiers in Artificial Intelligence and Applications, Vol. 267, IOS Press, 2014, pp. 105-118. doi:10.3233/978-1-61499-438-1-105.

[11] C. Chui and M. Grüninger, Merging the DOLCE and PSL upper ontologies, in: KEOD 2014 - Proceedings of the International Conference on Knowledge Engineering and Ontology Development, Rome, Italy, 21-24 October, 2014, J. Filipe, J.L.G. Dietz and D. Aveiro, eds, SciTePress, 2014, pp. 16-26. doi:10.5220/0005027100160026.

[12] M. Damova, A. Kiryakov, M. Grinberg, M.K. Bergman, F. Giasson and K. Simov, Creation and integration of reference ontologies for efficient lod management, in: Semi-Automatic Ontology Development: Processes and Resources, IGI Global, 2012, pp. 162-199. doi:10. 4018/978-1-4666-0188-8.ch007.

[13] M. Damova, A. Kiryakov, K.I. Simov and S. Petrov, Mapping the central LOD ontologies to PROTON upper-level ontology, in: Proceedings of the 5th International Workshop on Ontology Matching (OM-2010), Shanghai, China, November 7, 2010, P. Shvaiko, J. Euzenat, F. Giunchiglia, H. Stuckenschmidt, M. Mao and I.F. Cruz, eds, CEUR Workshop Proceedings, Vol. 689, CEUR-WS.org, 2010, http://ceurws.org/Vol-689/om2010_Tpaper6.pdf.

[14] J. David, J. Euzenat, F. Scharffe and C.T. dos Santos, The alignment API 4.0, Semantic Web 2(1) (2011), 3-10. doi:10.3233/SW-20110028.

[15] R. de Almeida Falbo, M.P. Barcellos, J.C. Nardi and G. Guizzardi, Organizing ontology design patterns as ontology pattern languages, in: The Semantic Web: Semantics and Big Data, 10th International Conference, ESWC 2013, Montpellier, France, May 26-30, 2013, Proceedings, P. Cimiano, Ó. Corcho, V. Presutti, L. Hollink and S. Rudolph, eds, Lecture Notes in Computer Science, Vol. 7882, Springer, 2013, pp. 61-75. doi:10.1007/978-3-642-38288-8_5.

[16] S. de Cesare and C. Partridge, BORO as a foundation to enterprise ontology, J. Inf. Syst. 30(2) (2016), 83-112. doi:10.2308/isys-51428.

[17] G. de Melo, F.M. Suchanek and A. Pease, Integrating YAGO into the suggested upper merged ontology, in: 20th IEEE International Conference on Tools with Artificial Intelligence (ICTAI 2008), Dayton, Ohio, USA, November 3-5, 2008, Vol. 1, IEEE Computer Society, 2008, pp. 190-193. doi:10.1109/ICTAI.2008.34. 
[18] J. Euzenat and P. Shvaiko, Ontology Matching, 2nd edn, Springer, 2013.

[19] C.M. Fonseca, D. Porello, G. Guizzardi, J.P.A. Almeida and N. Guarino, Relations in ontology-driven conceptual modeling, in: Conceptual Modeling - 38th International Conference, ER 2019, Salvador, Brazil, November 4-7, 2019, Proceedings, A.H.F. Laender, B. Pernici, E. Lim and J.P.M. de Oliveira, eds, Lecture Notes in Computer Science, Vol. 11788, Springer, 2019, pp. 28-42. doi:10.1007/978-3-03033223-5_4.

[20] A. Gangemi, N. Guarino, C. Masolo and A. Oltramari, Restructuring WordNet's top-level, AI Magazine 40 (2002), $235-244$.

[21] A. Gangemi, N. Guarino, C. Masolo and A. Oltramari, Sweetening WORDNET with DOLCE, AI Mag. 24(3) (2003), 13-24. doi:10.1609/ aimag.v24i3.1715.

[22] A. Gangemi, N. Guarino, C. Masolo, A. Oltramari and L. Schneider, Sweetening ontologies with DOLCE, in: Knowledge Engineering and Knowledge Management. Ontologies and the Semantic Web, 13th International Conference, EKAW 2002, Siguenza, Spain, October 1-4, 2002, Proceedings, A. Gómez-Pérez and V.R. Benjamins, eds, Lecture Notes in Computer Science, Vol. 2473, Springer, 2002 , pp. 166-181. doi:10.1007/3-540-45810-7_18.

[23] A. Gangemi and P. Mika, Understanding the semantic web through descriptions and situations, in: On the Move to Meaningful Internet Systems 2003: CoopIS, DOA, and ODBASE, R. Meersman, Z. Tari and D.C. Schmidt, eds, Springer Berlin Heidelberg, Berlin, Heidelberg, 2003, pp. 689-706. ISBN 978-3-540-39964-3. doi:10.1007/978-3-540-39964-3_44.

[24] A. Gangemi, R. Navigli and P. Velardi, The OntoWordNet project: Extension and axiomatization of conceptual relations in WordNet, in: On the Move to Meaningful Internet Systems 2003: CoopIS, DOA, and ODBASE - OTM Confederated International Conferences, CoopIS, DOA, and ODBASE 2003, Catania, Sicily, Italy, November 3-7, 2003, R. Meersman, Z. Tari and D.C. Schmidt, eds, Lecture Notes in Computer Science, Vol. 2888, Springer, 2003, pp. 820-838. doi:10.1007/978-3-540-39964-3_52.

[25] A. Gangemi, A.G. Nuzzolese, V. Presutti, F. Draicchio, A. Musetti and P. Ciancarini, Automatic typing of DBpedia entities, in: The Semantic Web - ISWC 2012 - 11th International Semantic Web Conference, Boston, MA, USA, November 11-15, 2012, Proceedings, Part I, P. Cudré-Mauroux, J. Heflin, E. Sirin, T. Tudorache, J. Euzenat, M. Hauswirth, J.X. Parreira, J. Hendler, G. Schreiber, A. Bernstein and E. Blomqvist, eds, Lecture Notes in Computer Science, Vol. 7649, Springer, 2012, pp. 65-81. doi:10.1007/978-3-642-35176-1_5.

[26] M.R. Genesereth, R.E. Fikes et al., Knowledge Interchange Format, Version 3.0 Reference Manual, Technical report, Logic-92-1, Stanford University, Stanford, CA, USA, 1992, citeseer.ist.psu.edu/genesereth92knowledge.html.

[27] P. Grenon, BFO in a Nutshell: A Bi-Categorial Axiomatization of BFO and Comparison with DOLCE, Leipzig, 2003.

[28] M. Gruninger, C. Chui and M. Katsumi, Upper ontologies in COLORE, in: Proceedings of the Joint Ontology Workshops 2017 Episode 3: The Tyrolean Autumn of Ontology, Bozen-Bolzano, Italy, September 21-23, 2017, S. Borgo, O. Kutz, F. Loebe, F. Neuhaus, K. Adrian, M. Antovic, V. Basile, M. Boeker, D. Calvanese, T. Caselli, G. Colombo, R. Confalonieri, L. Daniele, J. Euzenat, A. Galton, D. Gromann, M.M. Hedblom, H. Herre, I. Hinterwaldner, A. Janes, L. Jansen, K. Krois, A. Lieto, C. Masolo, R. Peñaloza, D. Porello, D.P. Radicioni, E.M. Sanfilippo, D. Schober, R. Stufano and A. Vizedom, eds, CEUR Workshop Proceedings, Vol. 2050, CEUR-WS.org, 2017.

[29] M. Grüninger and C. Menzel, The process specification language (PSL) theory and applications, AI Mag. 24(3) (2003), 63-74. doi:10.1609/ aimag.v24i3.1719.

[30] N. Guarino, Formal Ontology in Information Systems: Proceedings of the 1st International Conference, Trento, Italy, June 6-8, 1998, 1st edn, IOS Press, NLD, 1998. ISBN 9051993994.

[31] N. Guarino and C.A. Welty, An overview of OntoClean, in: Handbook on Ontologies, S. Staab and R. Studer, eds, International Handbooks on Information Systems, Springer, 2004, pp. 151-172.

[32] R. Guha and D. Lenat, Cyc: A midterm report, in: Readings in Knowledge Acquisition and Learning, 1993 , pp. 839-866.

[33] G. Guizzardi, Ontological foundations for structural conceptual models, PhD thesis, University of Twente, Enschede, The Netherlands, Enschede, 2005.

[34] G. Guizzardi, The role of foundational ontologies for conceptual modeling and domain ontology representation, in: 7th International Baltic Conference on Databases and Information Systems, 2006, pp. 17-25. doi:10.1109/DBIS.2006.1678468.

[35] G. Guizzardi, Ontology, ontologies and the "I" of FAIR, Data Intelligence 2(1-2) (2020), 181-191. doi:10.1162/dint_a_00040.

[36] G. Guizzardi, C.M. Fonseca, A.B. Benevides, J.P.A. Almeida, D. Porello and T.P. Sales, Endurant types in ontology-driven conceptual modeling: Towards OntoUML 2.0, in: Conceptual Modeling - 37th International Conference , ER 2018, Xi'an, China, October 22-25, 2018, Proceedings, J. Trujillo, K.C. Davis, X. Du, Z. Li, T.W. Ling, G. Li and M. Lee, eds, Lecture Notes in Computer Science, Vol. 11157 , Springer, 2018, pp. 136-150. doi:10.1007/978-3-030-00847-5_12.

[37] G. Guizzardi, G. Wagner, J.P.A. Almeida and R.S.S. Guizzardi, Towards ontological foundations for conceptual modeling: The unified foundational ontology (UFO) story, Appl. Ontology 10(3-4) (2015), 259-271. doi:10.3233/AO-150157.

[38] H. Herre, B. Heller, P. Burek, R. Hoehndorf, F. Loebe and H. Michalek, General formal ontology (GFO): A foundational ontology integrating objects and processes, in: Res. Group Ontologies in Medicine, 2007.

[39] P. Jain, P.Z. Yeh, K. Verma, R.G. Vasquez, M. Damova, P. Hitzler and A.P. Sheth, Contextual ontology alignment of LOD with an upper ontology: A case study with proton, in: The Semantic Web: Research and Applications - 8th Extended Semantic Web Conference, ESWC 2011 Heraklion, Crete, Greece, May 29-June 2, 2011, Proceedings, Part I, G. Antoniou, M. Grobelnik, E.P.B. Simperl, B. Parsia, D. Plexousakis, P.D. Leenheer and J.Z. Pan, eds, Lecture Notes in Computer Science, Vol. 6643, Springer, 2011, pp. 80-92. doi:10.1007/978-3642-21034-1_6.

[40] M. Kamel, D. Schmidt, C. Trojahn and R. Vieira, Hypernym relation extraction for establishing subsumptions: Preliminary results on matching foundational ontologies, in: Proceedings of the 14th International Workshop on Ontology Matching Co-Located with the 18th International Semantic Web Conference (ISWC 2019), Auckland, New Zealand, October 26, 2019, 2019, pp. 36-40, http://ceur-ws.org/Vol2536/om2019_STpaper1.pdf. 
[41] C.M. Keet, The use of foundational ontologies in ontology development: An empirical assessment, in: The Semantic Web: Research and Applications - 8th Extended Semantic Web Conference, ESWC 2011, Heraklion, Crete, Greece, May 29-June 2, 2011, Proceedings, Part I, G. Antoniou, M. Grobelnik, E.P.B. Simperl, B. Parsia, D. Plexousakis, P.D. Leenheer and J.Z. Pan, eds, Lecture Notes in Computer Science, Vol. 6643, Springer, 2011, pp. 321-335. doi:10.1007/978-3-642-21034-1_22.

[42] C.M. Keet and Z.C. Dawood, Addressing issues in foundational ontology mediation, in: KEOD 2013 - Proceedings of the International Conference on Knowledge Engineering and Ontology Development, Vilamoura, Algarve, Portugal, 19-22 September, 2013, J. Filipe and J.L.G. Dietz, eds, SciTePress, 2013, pp. 5-16. doi:10.5220/0004518000050016.

[43] Z.C. Khan and C.M. Keet, ONSET: Automated foundational ontology selection and explanation, in: Knowledge Engineering and Knowledge Management - 18th International Conference, EKAW 2012, Galway City, Ireland, October 8-12, 2012, Proceedings, A. ten Teije, J. Völker, S. Handschuh, H. Stuckenschmidt, M. d'Aquin, A. Nikolov, N. Aussenac-Gilles and N. Hernandez, eds, Lecture Notes in Computer Science, Vol. 7603, Springer, 2012, pp. 237-251. doi:10.1007/978-3-642-33876-2_22.

[44] Z.C. Khan and C.M. Keet, Feasibility of automated foundational ontology interchangeability, in: Knowledge Engineering and Knowledge Management - 19th International Conference, EKAW 2014, Linköping, Sweden, November 24-28, 2014, Proceedings, K. Janowicz, S. Schlobach, P. Lambrix and E. Hyvönen, eds, Lecture Notes in Computer Science, Vol. 8876, Springer, 2014, pp. 225-237. doi:10.1007/ 978-3-319-13704-9_18.

[45] F. Leão, K. Revoredo and F.A. Baião, Extending WordNet with UFO foundational ontology, J. Web Semant. 57 (2019). doi:10.1016/j. websem.2019.02.002.

[46] D.B. Lenat and R.V. Guha, The evolution of CycL, the Cyc representation language, SIGART Bull. 2(3) (1991), 84-87. doi:10.1145/122296. 122308.

[47] M. Lesk, Automatic sense disambiguation using machine readable dictionaries: How to tell a pine cone from an ice cream cone, in: Proceedings of the 5th Annual International Conference on Systems Documentation, SIGDOC 1986, Toronto, Ontario, Canada, 1986, V. DeBuys, ed., ACM, 1986, pp. 24-26. doi:10.1145/318723.318728.

[48] J. Li, LOM: A lexicon-based ontology mapping tool, in: Proceedings of the Performance Metrics for Intelligent Systems (PerMIS), 2004.

[49] X. Liu, B. Cheng, J. Liao, P.M. Barnaghi, L. Wan and J. Wang, OMI-DL: An ontology matching framework, IEEE Trans. Serv. Comput. 9(4) (2016), 580-593. doi:10.1109/TSC.2015.2410794.

[50] V. Mascardi, V. Cordì and P. Rosso, A comparison of upper ontologies, in: WOA 2007: Dagli Oggetti agli Agenti. 8th AI*IA/TABOO Joint Workshop "From Objects to Agents": Agents and Industry: Technological Applications of Software Agents, Genova, Italy, 24-25 September 2007, M. Baldoni, A. Boccalatte, F.D. Paoli, M. Martelli and V. Mascardi, eds, Seneca Edizioni Torino, 2007, pp. 55-64.

[51] V. Mascardi, A. Locoro and P. Rosso, Automatic ontology matching via upper ontologies: A systematic evaluation, IEEE Trans. Knowl. Data Eng. 22(5) (2010), 609-623. doi:10.1109/TKDE.2009.154.

[52] P. Mika, D. Oberle, A. Gangemi and M. Sabou, Foundations for service ontologies: Aligning OWL-S to dolce, in: Proceedings of the 13th International Conference on World Wide Web, WWW 2004, New York, NY, USA, May 17-20, 2004, S.I. Feldman, M. Uretsky, M. Najork and C.E. Wills, eds, ACM, 2004, pp. 563-572. doi:10.1145/988672.988748.

[53] T. Mikolov, I. Sutskever, K. Chen, G.S. Corrado and J. Dean, Distributed representations of words and phrases and their compositionality, in: Advances in Neural Information Processing Systems 26: 27th Annual Conference on Neural Information Processing Systems 2013, Lake Tahoe, Nevada, United States, December 5-8, 2013, Proceeedings, C.J.C. Burges, L. Bottou, Z. Ghahramani and K.Q. Weinberger, eds, 2013, pp. 3111-3119.

[54] G.A. Miller, WordNet: A lexical database for English, Commun. ACM 38(11) (1995), 39-41. doi:10.1145/219717.219748.

[55] R. Mizoguchi, YAMATO: Yet another more advanced top-level ontology, in: Proceedings of the Sixth Australasian Ontology Workshop, 2010, pp. 1-16.

[56] L.S. Muñoz and M. Grüninger, Verifying and mapping the mereotopology of upper-level ontologies, in: Proceedings of the 8th International Joint Conference on Knowledge Discovery, Knowledge Engineering and Knowledge Management (IC3K 2016) - Volume 2: KEOD, Porto, Portugal, November 9-11, 2016, A.L.N. Fred, J.L.G. Dietz, D. Aveiro, K. Liu, J. Bernardino and J. Filipe, eds, SciTePress, 2016, pp. 31-42. doi: $10.5220 / 0006052100310042$.

[57] I. Niles and A. Pease, Towards a standard upper ontology, in: 2nd International Conference on Formal Ontology in Information Systems, FOIS 2001, Ogunquit, Maine, USA, October 17-19, 2001, Proceedings, ACM, 2001, pp. 2-9. doi:10.1145/505168.505170.

[58] I. Niles and A. Pease, Linking lexicons and ontologies: Mapping WordNet to the suggested upper merged ontology, in: Proc. of the Inter. Conf. on Knowledge Engineering, 2003, pp. 412-416.

[59] D. Oberle, A. Ankolekar, P. Hitzler, P. Cimiano, M. Sintek, M. Kiesel, B. Mougouie, S. Baumann, S. Vembu and M. Romanelli, DOLCE ergo SUMO: On foundational and domain models in the SmartWeb integrated ontology (SWIntO), J. Web Semant. 5(3) (2007), 156-174. doi:10.1016/j.websem.2007.06.002.

[60] N.F. Padilha, F.A. Baião and K. Revoredo, Alignment patterns based on unified foundational ontology, in: Proceedings of Joint V Seminar on Ontology Research in Brazil and VII International Workshop on Metamodels, Ontologies and Semantic Technologies, Recife, Brazil, September 19-21, 2012, A. Malucelli and M.P. Bax, eds, CEUR Workshop Proceedings, Vol. 938, CEUR-WS.org, 2012, pp. 48-59.

[61] H. Paulheim and A. Gangemi, Serving DBpedia with DOLCE - More than just adding a cherry on top, in: The Semantic Web - ISWC 2015 14th International Semantic Web Conference, Bethlehem, PA, USA, October 11-15, 2015, Proceedings, Part I, M. Arenas, Ó. Corcho, E. Simperl, M. Strohmaier, M. d'Aquin, K. Srinivas, P. Groth, M. Dumontier, J. Heflin, K. Thirunarayan and S. Staab, eds, Lecture Notes in Computer Science, Vol. 9366, Springer, 2015, pp. 180-196. doi:10.1007/978-3-319-25007-6_11.

[62] A. Pease, Ontology: A Practical Guide, Articulate Software Press, Angwin, CA, 2011.

[63] A. Pease and C. Benzmüller, Sigma: An integrated development environment for logical theories, AI Comm. 26 (2013), 9-97. 
[64] A. Pease, G. Sutcliffe, N. Siegel and S. Trac, Large theory reasoning with SUMO at CASC, AI Communications, Special Issue on Practical Aspects of Automated Reasoning 23(2-3) (2010), 137-144.

[65] E. Prestes, J.L. Carbonera, S.R. Fiorini, V.A.M. Jorge, M. Abel, R. Madhavan, A. Locoro, P.J.S. Gonçalves, M.E. Barreto, M.K. Habib, A. Chibani, S. Gérard, Y. Amirat and C. Schlenoff, Towards a core ontology for robotics and automation, Robotics Auton. Syst. 61(11) (2013), 1193-1204. doi:10.1016/j.robot.2013.04.005.

[66] F. Probst, Ontological analysis of observations and measurements, in: Geographic Information Science, 4th International Conference, GIScience 2006, Münster, Germany, September 20-23, 2006, Proceedings, M. Raubal, H.J. Miller, A.U. Frank and M.F. Goodchild, eds, Lecture Notes in Computer Science, Vol. 4197, Springer, 2006, pp. 304-320. doi:10.1007/11863939_20.

[67] S. Reed and D. Lenat, Mapping ontologies into Cyc, in: Proc. of the Workshop on Ontologies for the Semantic Web, 2002, pp. 1-6.

[68] A. Scherp, C. Saathoff, T. Franz and S. Staab, Designing core ontologies, Appl. Ontology 6(3) (2011), 177-221. doi:10.3233/AO-20110096.

[69] D. Schmidt, R. Basso, C. Trojahn and R. Vieira, Matching domain and top-level ontologies via OntoWordNet, in: Proceedings of the 12th International Workshop on Ontology Matching Co-Located with the 16th International Semantic Web Conference (ISWC 2017), Vienna, Austria, October 21, 2017, P. Shvaiko, J. Euzenat, E. Jiménez-Ruiz, M. Cheatham and O. Hassanzadeh, eds, CEUR Workshop Proceedings, Vol. 2032, CEUR-WS.org, 2017, pp. 225-226, http://ceur-ws.org/Vol-2032/om2017_poster10.pdf.

[70] D. Schmidt, R. Basso, C. Trojahn and R. Vieira, Matching domain and top-level ontologies exploring word sense disambiguation and word embedding, in: Emerging Topics in Semantic Technologies - ISWC 2018 Satellite Events [Best Papers from 13 of the Workshops Co-Located with the ISWC 2018 Conference], E. Demidova, A. Zaveri and E. Simperl, eds, Studies on the Semantic Web, Vol. 36, IOS Press, 2018, pp. 27-38. doi:10.3233/978-1-61499-894-5-27.

[71] D. Schmidt, C.T. dos Santos and R. Vieira, Analysing top-level and domain ontology alignments from matching systems, in: Proceedings of the 11th International Workshop on Ontology Matching Co-Located with the 15th International Semantic Web Conference (ISWC 2016), Kobe, Japan, October 18, 2016, P. Shvaiko, J. Euzenat, E. Jiménez-Ruiz, M. Cheatham, O. Hassanzadeh and R. Ichise, eds, CEUR Workshop Proceedings, Vol. 1766, CEUR-WS.org, 2016, pp. 13-24.

[72] D. Schmidt, A. Pease, C. Trojahn and R. Vieira, Aligning conference ontologies with SUMO: A report on manual alignment via WordNet, in: Proceedings of the Joint Ontology Workshops 2019 Episode V: The Styrian Autumn of Ontology, Graz, Austria, September 23-25, 2019, 2019, http://ceur-ws.org/Vol-2518/paper-FOUST10.pdf.

[73] D. Schmidt, C. Trojahn and R. Vieira, Matching BFO, DOLCE, GFO and SUMO: An evaluation of OAEI 2018 matching systems, in: Proceedings of the XII Seminar on Ontology Research in Brazil and III Doctoral and Masters Consortium on Ontologies, Porto Alegre, Brazil, September 2nd-5th, 2019, J.P.A. Almeida, M. Bax, R. Berardi and F.A. Baião, eds, CEUR Workshop Proceedings, Vol. 2519, CEUR-WS.org, 2019.

[74] D. Schmidt, C. Trojahn, R. Vieira and M. Kamel, Validating top-level and domain ontology alignments using WordNet, in: Proceedings of the IX ONTOBRAS Brazilian Ontology Research Seminar, Curitiba, Brazil, October 3rd, 2016, R.M.A. Baracho, S. Isotani and M.B. Almeida, eds, CEUR Workshop Proceedings, Vol. 1862, CEUR-WS.org, 2016, pp. 119-130.

[75] S. Schulz, The role of foundational ontologies for preventing bad ontology design, in: Proceedings of the Joint Ontology Workshops 2018 Episode IV: The South African Spring Co-Located with the 10th International Conference on Formal Ontology in Information Systems (FOIS 2018), Cape Town, South Africa, September 17-18, 2018, L. Jansen, D.P. Radicioni and D. Gromann, eds, CEUR Workshop Proceedings, Vol. 2205, CEUR-WS.org, 2018.

[76] S. Semy, M. Pulvermacher and L. Obrst, Toward the use of an upper ontology for U.S. government and U.S. military domains: An evaluation, Technical report, MTR 04B0000063, The MITRE Corporation, 2004.

[77] S. Seppälä, Mapping WordNet to the basic formal ontology using the KYOTO ontology, in: Proceedings of the International Conference on Biomedical Ontology, ICBO 2015, Lisbon, Portugal, July 27-30, 2015, F.M. Couto and J. Hastings, eds, CEUR Workshop Proceedings, Vol. 1515, CEUR-WS.org, 2015.

[78] S. Seppälä, B. Smith and W. Ceusters, Applying the realism-based ontology-versioning method for tracking changes in the basic formal ontology, in: Formal Ontology in Information Systems - Proceedings of the Eighth International Conference, FOIS 2014, Rio de Janeiro, Brazil, September, 22-25, 2014, P. Garbacz and O. Kutz, eds, Frontiers in Artificial Intelligence and Applications, Vol. 267, IOS Press, 2014, pp. 227-240. doi:10.3233/978-1-61499-438-1-227.

[79] A.P. Seyed, BFO/DOLCE primitive relation comparison, in: Nature Proceedings, 2009.

[80] P. Shvaiko and J. Euzenat, Ten challenges for ontology matching, in: On the Move to Meaningful Internet Systems: OTM 2008, R. Meersman and Z. Tari, eds, Springer Berlin Heidelberg, Berlin, Heidelberg, 2008, pp. 1164-1182. ISBN 978-3-540-88873-4. doi:10.1007/978-3-54088873-4_18.

[81] V.D.S. Silva, A. Freitas and S. Handschuh, Word tagging with foundational ontology classes: Extending the WordNet-DOLCE mapping to verbs, in: Knowledge Engineering and Knowledge Management - 20th International Conference, EKAW 2016, Bologna, Italy, November 19-23, 2016, Proceedings, E. Blomqvist, P. Ciancarini, F. Poggi and F. Vitali, eds, Lecture Notes in Computer Science, Vol. 10024, 2016, pp. 593-605. doi:10.1007/978-3-319-49004-5_38.

[82] V.S. Silva, M.L.M. Campos, J.C.P. Silva and M.C. Cavalcanti, An approach for the alignment of biomedical ontologies based on foundational ontologies, J. Inf. Data Manag. 2(3) (2011), 557-572.

[83] G. Stapleton, J. Howse, A. Bonnington and J. Burton, A vision for diagrammatic ontology engineering, in: Proceedings of the International Workshop on Visualizations and User Interfaces for Knowledge Engineering and Linked Data Analytics Co-Located with 19th International Conference on Knowledge Engineering and Knowledge Management, VISUAL@EKAW 2014, Linköping, Sweden, November 24, 2014, V. Ivanova, T. Kauppinen, S. Lohmann, S. Mazumdar, C. Pesquita and K. Xu, eds, CEUR Workshop Proceedings, Vol. 1299, CEURWS.org, 2014, pp. 1-13. 
[84] R. Stevens, P. Lord, J. Malone and N. Matentzoglu, Measuring expert performance at manually classifying domain entities under upper ontology classes, J. Web Semant. 57 (2019). doi:10.1016/j.websem.2018.08.004.

[85] L. Temal, A. Rosier, O. Dameron and A. Burgun, Mapping BFO and DOLCE, in: MEDINFO 2010 - Proceedings of the 13th World Congress on Medical Informatics, Cape Town, South Africa, September 12-15, 2010, C. Safran, S.R. Reti and H.F. Marin, eds, Studies in Health Technology and Informatics, Vol. 160, IOS Press, 2010, pp. 1065-1069. doi:10.3233/978-1-60750-588-4-1065.

[86] I. Terziev, A. Kiryakov and D. Manov, Base Upper-level Ontology (BULO) Guidance, Deliverable 1.8.1, SEKT project, D1.8.1, 2005.

[87] É. Thiéblin, O. Haemmerlé, N. Hernandez and C. Trojahn, Survey on complex ontology matching, Semantic Web 11(4) (2020), 689-727. doi:10.3233/SW-190366.

[88] C. Trojahn, Is your data 6-star?, in: Proceedings of the ISWC 2020 Demos and Industry Tracks: From Novel Ideas to Industrial Practice CoLocated with 19th International Semantic Web Conference (ISWC 2020), Globally Online, November 1-6, 2020 (UTC), CEUR-WS.org, 2020, pp. 286-290.

[89] M. Uschold and D. McComb, Introduction to Gist, White paper, Semantic Arts, 2013.

[90] M. Verdonck, T.P. Sales and F. Gailly, A comparative illustration of foundational ontologies: BORO and UFO, in: Proceedings of the Joint Ontology Workshops 2018 Episode IV: The South African Spring Co-Located with the 10th International Conference on Formal Ontology in Information Systems (FOIS 2018), Cape Town, South Africa, September 17-18, 2018, L. Jansen, D.P. Radicioni and D. Gromann, eds, CEUR Workshop Proceedings, Vol. 2205, CEUR-WS.org, 2018. 